\title{
Reducing Cardiovascular Disease Risk in Women Beyond Statin Therapy: New Insights 2020
}

\author{
Lori Mosca, MD, MPH, PhD, ${ }^{1}$ Ann Marie Navar, MD, $\mathrm{PhD},{ }^{2}$ and Nanette Kass Wenger, $\mathrm{MD}^{3}$
}

\begin{abstract}
Management of residual and persistent cardiovascular disease (CVD) risk among statin-treated individuals has emerged as an important preventive strategy. The purpose of this article is to review the unique landscape of CVD in women and relevant prior prevention trials, and to discuss how the recent results of the Reduction of Cardiovascular Events with Icosapent Ethyl-Intervention Trial (REDUCE-IT) might apply to the contemporary management of CVD risk among statin-treated women. Women have unique risk factors that may impact CVD and its prevention. Historically, women have been underrepresented in CVD trials, posing a challenge to development of clinical recommendations for women. Low-density lipoprotein cholesterol-targeting treatments have demonstrated CVD risk reduction, with comparable effects in both sexes. In contrast, triglyceridelowering treatments (niacin, fenofibrate, and omega-3 fatty acids) have reported mixed findings for CVD risk reduction. Recent clinical trials of combination omega-3 fatty acids (docosahexaenoic acid/eicosapentaenoic acid [EPA]) have not found significant CVD risk reduction. The recently published REDUCE-IT study found that icosapent ethyl, an EPA-only omega-3 fatty acid, in combination with statins, significantly reduced CVD events in high-risk patients. The icosapent ethyl group had a significantly lower occurrence of the primary composite CVD endpoint (17.2\%) than the placebo group (22.0\%; hazard ratio $0.75 ; 95 \%$ confidence interval $0.68-0.83 ; p<0.001)$. CVD risk reduction with icosapent ethyl treatment was comparable between women and men ( $p$ for interaction, 0.33). Data from REDUCE-IT suggest women benefit similarly to men with respect to icosapent ethyl, a novel therapy for prevention of CVD.
\end{abstract}

Keywords: cardiovascular disease, eicosapentaenoic acid, icosapent ethyl, sex differences, women

\section{Introduction}

D ESPITE CONSIDERABLE PROGRESS in reducing the risk of cardiovascular disease (CVD) with evidence-based therapies such as statins, CVD remains the leading cause of death among women, with substantial human and economic toll. ${ }^{1}$ The fact that sex-based differences in biology can impact health and disease has been recognized ${ }^{2}$; however, historically, women have been underrepresented in CVD trials, which has led to challenges in development of clinical guidelines to reduce CVD risk in women., ${ }^{3,4}$

Addressing residual CVD risk among statin-treated individuals who have controlled levels of low-density lipoprotein cholesterol (LDL-C) has broadly been recognized as an important clinical strategy. However, studies targeting lipids beyond LDL-C, such as triglycerides (TG), using fibrates, niacin, or combination omega-3 fatty acids (eicosapentaenoic acid [EPA] and docosahexaenoic acid [DHA]), have yielded disappointing or inconsistent results; some have failed to show clinical benefit in combination with a statin overall, ${ }^{5-8}$ and one even suggested potential harm among women. ${ }^{6}$

Recently, addition of an omega-3 fatty acid (EPA; icosapent ethyl) to statins in high-risk individuals demonstrated reductions in major CVD events in the Reduction of Cardiovascular Events with Icosapent Ethyl-Intervention Trial (REDUCE-IT). ${ }^{9}$ While these findings are promising, it is

\footnotetext{
${ }^{1}$ Division of Cardiology, Department of Medicine, Columbia University Irving Medical Center, New York, New York, USA.

${ }^{2}$ Department of Medicine, Division of Cardiology, Duke Clinical Research Institute, Durham, North Carolina, USA.

${ }^{3}$ Department of Cardiology, Emory University School of Medicine; Emory Heart and Vascular Center; Emory Women's Heart Center, Atlanta, Georgia, USA.
} 
important to examine the efficacy and safety of icosapent ethyl in women, and to place results in the context of other therapies proven to prevent CVD events. The purpose of this article is to review the unique experiences of CVD in women and relevant prior CVD trials in women, and to discuss how the results of REDUCE-IT might apply to the contemporary management of residual CVD risk in women.

\section{CVD Experience in Women}

Despite substantial reductions in CVD death over the past 40 years, there has been a concerning upward trend in mortality over the most recent decade (Fig. 1). ${ }^{1}$ While rates of decline in CVD mortality have diminished in recent decades for both men and women $<55$ years of age, the rates of decline have been consistently lower for women than for men. ${ }^{1}$

Recent trends in CVD death may be related, in part, to the rise in diabetes, with the overall prevalence of diagnosed diabetes mellitus having increased from $5.0 \%$ in $1999-2000$ to $7.8 \%$ in $2009-2010 .{ }^{1}$ In women, the prevalence estimates for diagnosed diabetes, undiagnosed diabetes, and prediabetes are $8.9 \%, 2.8 \%$, and $31.3 \%$, respectively, based on The National Health and Nutrition Examination Survey (NHANES) 2013-2016 data. ${ }^{1}$ The relative risk of CVD in patients with diabetes versus without diabetes is more pronounced in women (risk ratio [RR] 3.57) than in men (RR 1.93). ${ }^{10}$

The greater association between diabetes and CVD risk in women compared with men may be due to the more deleterious effects of diabetes and lipids on blood pressure in women $^{11,12}$; the interplay between lipids and insulin resistance may contribute to endothelial dysfunction, which has the potential to impact hypertension and diabetes risk. ${ }^{13-17}$

Women develop CVD at an older age compared with men, and factors such as diabetes and hypertriglyceridemia appear to better predict the risk of CVD in women compared with men. ${ }^{18,19}$ Women's sex hormones may play a role in CVD risk; before menopause, women have greater subcutaneous adipose tissue and greater TG clearance, but this is reduced postmenopause, and associated with increased TG and metabolic syndrome, ${ }^{20}$ which may both contribute to elevated CVD risk.
Beyond lipids, a number of other uniquely important factors may have implications for CVD risk in women, including psychosocial risk factors such as depression, poor sleep quality, inadequate hours of sleep, complications of pregnancy, poor adherence, and fear of side effects. ${ }^{10,21-24}$ Risk factors that cause stress, such as depression and posttraumatic stress disorder, as well as social isolation and greater family responsibilities, are more prevalent in women than men, and have demonstrated robust associations with CVD. ${ }^{10,25}$ Thus, while CVD risk management should focus on areas where intervention has been proven to ameliorate risk, it is important to consider the potential impact of psychosocial risk factors on CVD risk and on adherence to proven therapies.

\section{Suboptimal preventive care in women}

CVD risk may be underestimated in women, posing a barrier to optimal preventive care. An American Heart Association (AHA) national survey of 500 physicians (primary care physicians, obstetricians/gynecologists, and cardiologists) found that the main driver of CVD prevention was proper assessment of baseline risk; however, women, despite similar calculated risk to men, were more likely to be assigned a lower risk category. ${ }^{26}$ The American College of Cardiology (ACC)/AHA CVD risk calculator has been shown to underperform in women (especially young women), potentially causing patients and physicians to misjudge risk. ${ }^{27}$

Differences in the presentation and symptoms of CVD in women compared with men ${ }^{18,19}$ may further contribute to a misconception that women have a lower CVD risk. Even though women have a recurrence rate of myocardial infarction (MI) that is three times greater than men, ${ }^{28}$ women with acute coronary syndrome (ACS) or MI are less likely to undergo revascularization, receive appropriate medication, or receive cardiac rehabilitation than men. ${ }^{29-36}$

\section{Underrepresentation in clinical trials}

Historically, a major challenge in the management of CVD risk in women has been the lack of data compared with men owing to the lower enrollment rates for women in clinical
FIG. 1. Change in cardiovascular deaths in men and women since 1979. Reprinted with permission, Benjamin et al., (2019 American Heart Association, Inc. ${ }^{1}$

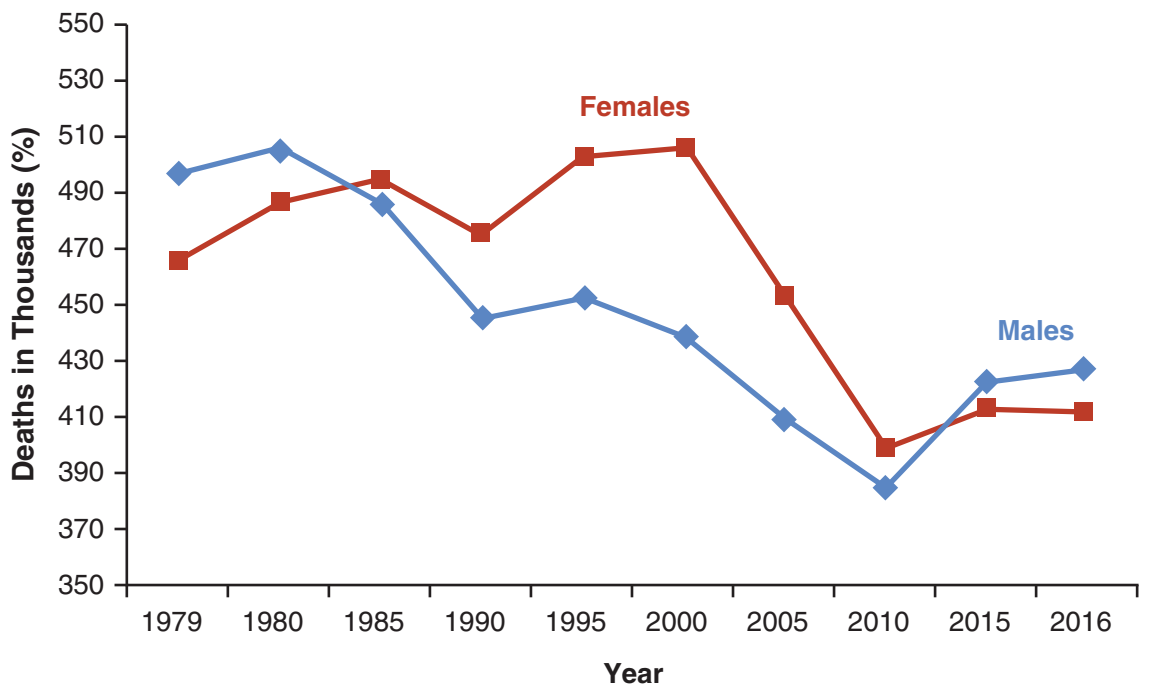


trials. Many reasons underlie the underrepresentation of women in CVD trials. Women tend to present with CVD on average 10 years later than men, ${ }^{34}$ which may contribute to exclusion from trials due to upper age limits ${ }^{3,37}$ as well as exclusions due to pregnancy-related concerns for younger women. Moreover, women may have logistical barriers to participation due to lack of transportation and/or caregiving responsibilities. $^{37,38}$

In an effort to address the sex-based disparities in research and clinical studies, the National Institutes of Health and the Institute of Medicine published recommendations to promote inclusion of women in clinical research and sex-/genderbased subpopulation analyses, and to make sex-specific data more readily available. ${ }^{2,39}$ Progress has been made, with recent CVD trials showing a trend in enrolling larger numbers of women. ${ }^{40}$ Critical evaluation of new and ongoing CVD outcome studies, with attention to sex-based differences, and the application of these findings to clinical practice may help to guide CVD management in women.

\section{Examining Sex-Based Differences in CVD Trials}

\section{Statin trials}

Analyses of sex-based differences in statin CVD outcome trials have generally found comparable outcomes between women and men, although sample sizes are typically powered to detect differences in treatment effect for the overall study population and may be insufficient for identifying statistically significant differences between sexes. ${ }^{38}$

The Justification for the Use of Statins in Prevention: an Intervention Trial Evaluating Rosuvastatin (JUPITER) ( $N=17,802 ; 38.2 \%$ women) is of interest, given the large population of women. ${ }^{41}$ This trial studied rosuvastatin for primary prevention of CVD events in women $\geq 60$ years of age and men $\geq 50$ years of age with LDL-C $<130 \mathrm{mg} / \mathrm{dL}{ }^{40}$ After 12 months, changes in LDL-C and TG were similar for women and men. A significant reduction versus placebo in the primary composite endpoint (MI, stroke, hospitalization for unstable angina, arterial revascularization, or CVD death) was reported for both men (hazard ratio [HR] $0.58 ; 95 \%$ confidence interval $[\mathrm{CI}] 0.45-0.73 ; p<0.001)$ and women (HR 0.54; 95\% CI 0.37-0.80; $p=0.002$ ), with no significant treatment-by-sex interaction $(p=0.80)$.

A meta-analysis of 11 randomized, double-blind, placebocontrolled CVD outcome trials of statins for secondary prevention found that statins were effective for prevention of CVD endpoints in women and men overall; stratification by sex found that statins did not achieve significance versus placebo for the endpoints of all-cause mortality and stroke in women. ${ }^{42}$ A systematic review of primary prevention studies supported that statins have benefits for primary prevention even among low-risk groups such as women. ${ }^{43}$

The largest meta-analysis comparing statin outcomes by sex (27 randomized clinical trials; $N=174,149 ; 26.8 \%$ women) found that, although women had a lower CVD risk than men, the proportional reduction in major vascular events with statin treatment among women was comparable to the reduction among men (adjusted $p=0.33$ for heterogeneity). ${ }^{44}$ Statin effects were comparable for women and men with a definite history of CVD (secondary prevention: adjusted $p=0.431$ for heterogeneity); however, the risk reduction among individuals with no known history of CVD was smaller in women (primary prevention: adjusted $p=0.02$ for heterogeneity). These data support that statins reduce major vascular events comparably in women and men with equivalent CVD risk.

\section{Nonstatin treatments for lowering $L D L-C$}

Add-on therapy to statins for further LDL-C reduction has emerged as an effective strategy for addressing residual CVD risk. The Improved Reduction of Outcomes: Vytorin Efficacy International Trial (IMPROVE-IT) $(N=18,144 ; 24.3 \%$ women) demonstrated that ezetimibe, in combination with simvastatin, reduced the primary composite endpoint (CVD death, nonfatal MI, rehospitalization for unstable angina, or coronary revascularization) in patients $>50$ years of age, who had been hospitalized within 10 days of randomization for ACS. ${ }^{4,46}$ After 7 years of follow-up, the HR for the reduction in CVD events for ezetimibe versus placebo was $0.88(95 \%$ CI 0.79-0.99) for women and 0.95 (95\% CI 0.90-1.01) for men, with no statistically significant difference between sexes $(p=0.26)$.

The Further Cardiovascular Outcomes Research with PCSK9 Inhibition in Subjects with Elevated Risk (FOURIER) study ( $N=27,564 ; 24.6 \%$ women) demonstrated that the PCSK9 inhibitor evolocumab, in combination with a statin, significantly reduced the primary composite endpoint (CVD death, MI, stroke, hospitalization for unstable angina, or coronary revascularization) in patients 40 to 85 years of age with clinically evident atherosclerotic CVD. A sex-based subanalysis found that the HR for the relative risk reduction in the primary endpoint was 0.81 (95\% CI 0.69-0.95) for women and 0.86 (95\% CI 0.80-0.94) for men, with no difference between sexes ( $p$ for heterogeneity was not significant). ${ }^{47}$ These data suggest that the benefits of ezetimibe and evolocumab as add-on therapy in statin-treated patients are comparable between women and men.

\section{Nonstatin treatments: beyond targeting $L D L-C$}

Beyond LDL-C, TG has been a long-standing theoretical therapeutic target for addressing residual CVD risk. However, high-profile trials of TG-lowering treatments have reported inconsistent findings overall and/or with respect to women (Table 1). ${ }^{5-9,48-54}$ Studies of the TG-lowering drug fenofibrate yielded mixed results. The Fenofibrate Intervention and Event Lowering in Diabetes (FIELD) study $(N=9,795 ; 37.3 \%$ women) randomized patients with type 2 diabetes who were not using a statin to fenofibrate or placebo. ${ }^{5}$ Improvements with fenofibrate relative to placebo were greater in women versus men for both LDL-C $(9.8 \%$ vs. $3.3 \%$ at study end, $p<0.001)$ and total cholesterol $(9.5 \%$ vs. $5.2 \%, p<0.001)$; changes in high-density lipoprotein cholesterol (HDL-C) and TG were similar for women and men. In the overall population, there was no significant improvement in the primary composite endpoint (HR 0.89; 95\% CI $0.75-1.05 ; p=0.16)$. Although the $p$-value for the interaction analysis was nonsignificant $(p=0.3)$, in subgroup analyses, total CVD events were reduced by $20 \%$ in women (HR 0.8; 95\% CI 0.64-0.99; $p=0.04$ ) and nonsignificantly by $8 \%$ in men (HR $0.92 ; 95 \%$ CI $0.81-1.05$; $p=0.2$ ). Among patients who did not have CVD at study entry, fenofibrate reduced the risk of total CVD events by 


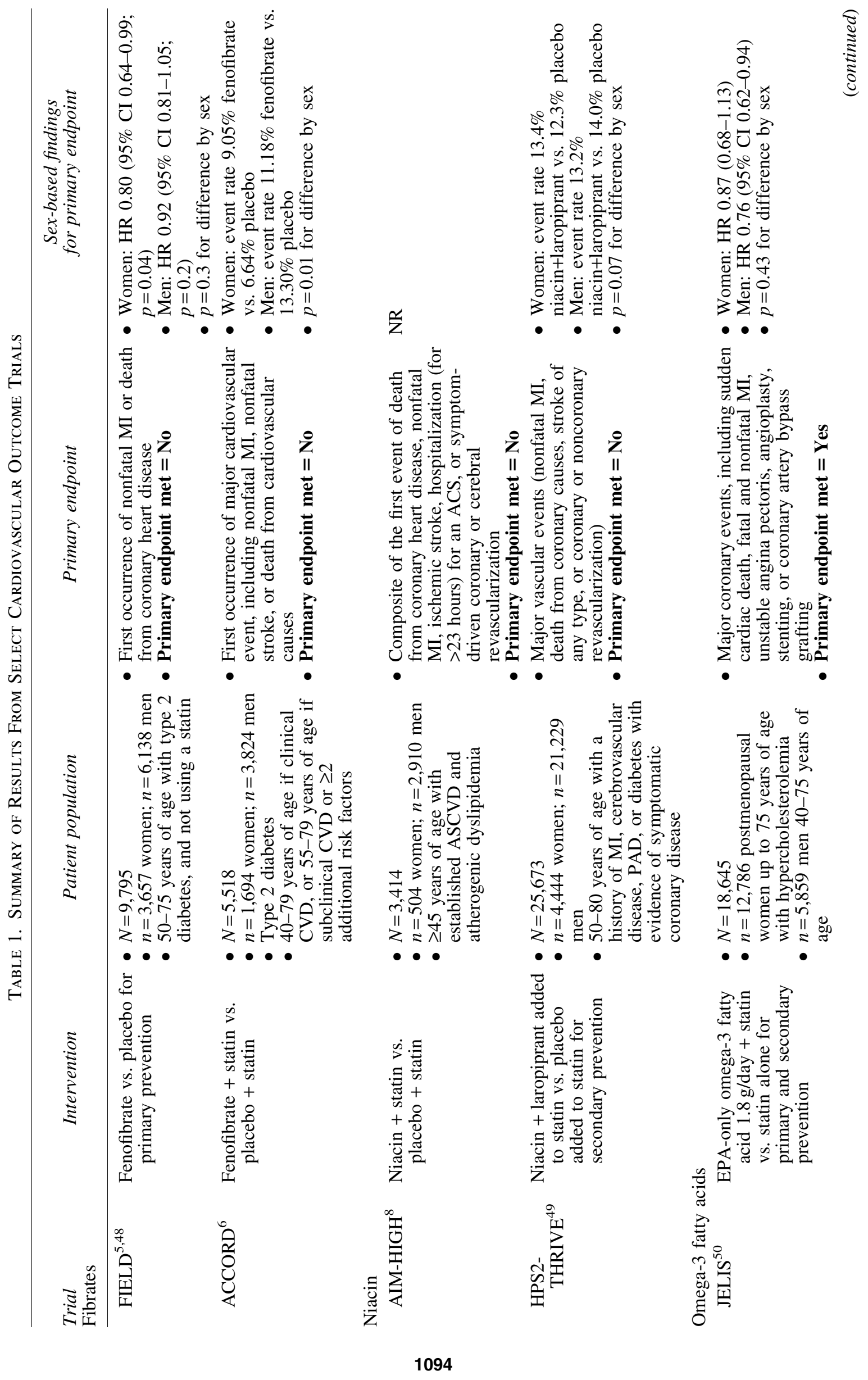




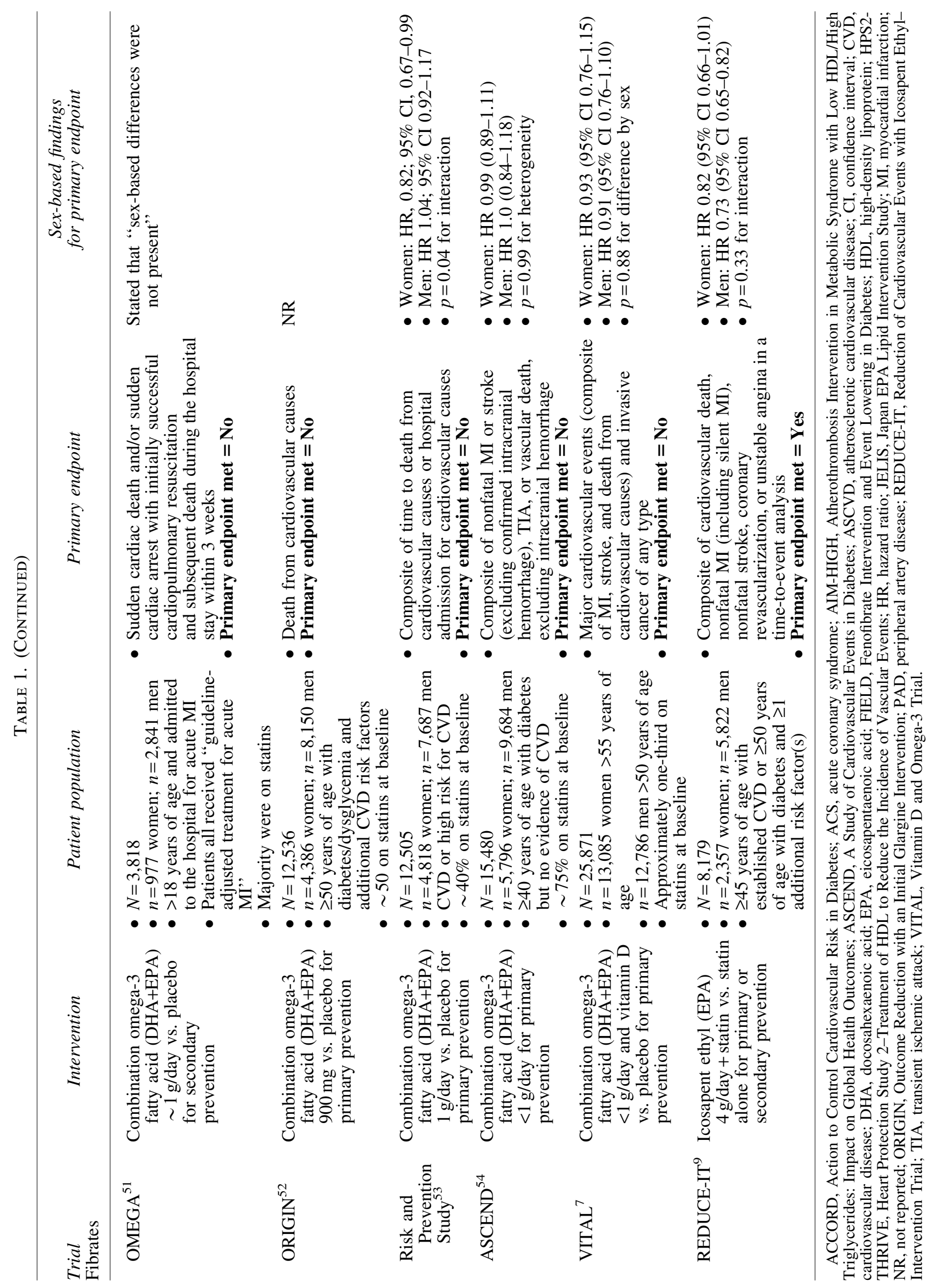


$26 \%$ in women and $16 \%$ in men (both $p=0.04$ ), with no significant interaction by sex $(p=0.45)$.

The Action to Control Cardiovascular Risk in Diabetes (ACCORD) study $(N=5,518 ; 30.7 \%$ women) found that addition of fenofibrate to a statin did not reduce the rate of CVD endpoints compared with statin alone in the majority of high-risk patients with diabetes mellitus. A significant interaction by sex favoring men was found $(p=0.01)$ : women had a higher CVD event rate with fenofibrate plus statin than with placebo plus statin $(9.1 \%$ vs. $6.6 \%)$, whereas men had a lower CVD event rate with fenofibrate plus statin than with placebo plus statin $(11.2 \%$ vs. $13.3 \%){ }^{6}$ These collective data show that fibrates did not reduce CVD risk overall, with potentially negative findings regarding effects in women.

Initial interest in niacin derived from the theory that drugs that increase HDL-C could potentially reduce CVD risk. ${ }^{55}$ Studies of niacin in the secondary prevention of CVD were highly anticipated, yet had disappointing findings. ${ }^{8,49}$ The Atherothrombosis Intervention in Metabolic Syndrome with Low HDL/High Triglycerides: Impact on Global Health Outcomes (AIM-HIGH) study $(N=3,414 ;<15 \%$ women) found that addition of niacin to statin treatment failed to reduce $C V D$ events in patients with atherosclerotic CVD and LDL-C $<70 \mathrm{mg} / \mathrm{dL}$ despite increases in HDL-C and decreases in TG. ${ }^{8}$

Subsequently, the Heart Protection Study 2-Treatment of HDL to Reduce the Incidence of Vascular Events (HPS2THRIVE) $(N=25,673 ;<18 \%$ women $)$ found that adding a combination of niacin with laropiprant to simvastatin \pm ezetimibe did not reduce major vascular events, and increased the risk of serious adverse events. ${ }^{49}$ A sex-based subanalysis of HPS2-THRIVE showed a trend $(p=0.07)$ toward worse CVD outcomes among niacin-treated women.

Investigations of omega-3 fatty acids for CVD risk reduction have reported mixed findings. The Japan EPA Lipid Intervention Study (JELIS), an open-label trial conducted in a Japanese population $(N=19,466 ; 69 \%$ women $)$ with hypercholesterolemia, randomized patients to EPA $600 \mathrm{mg}$ three times a day on a background of statin treatment or to statin treatment alone. ${ }^{50}$ The EPA group had a significant relative risk reduction of $19 \%$ in major CVD events compared with the control group (HR 0.81; 95\% CI 0.69-0.95; $p=0.011$ ) over 5 years. The CVD event rate was lower for women than men in both the control and EPA-treated groups. The HR for the reduction in CVD events with EPA versus placebo was 0.87 (95\% CI 0.68-1.13) for women and 0.76 (95\% CI $0.62-0.94)$ for men, with no interaction by sex $(p=0.43)$. EPA was associated with a significant improvement in secondary prevention of CVD events (HR 0.81; $95 \%$ CI $0.66-1.0 ; p=0.048)$, but did not reach statistical significance for primary prevention (HR $0.82 ; 95 \%$ CI $0.63-1.06$ ).

Subsequent omega-3 fatty acid CVD outcome trials investigating DHA+EPA combinations have failed to achieve their primary endpoints (Table 1). ${ }^{7,51-54}$ Among outcome trials of DHA+EPA that reported sex-based subanalyses, only the Risk and Prevention Study found a difference between sexes ( $p=0.04$ for interaction): a significantly lower rate of CVD events was found among women receiving DHA+EPA versus placebo (HR 0.82; 95\% CI 0.67-0.99), with no significant finding in men (HR 1.04; 95\% CI 0.92-1.17). ${ }^{53}$

The most recent combination omega-3 fatty acid outcome trial, the Vitamin D and Omega-3 Trial (VITAL) $(N=25,871$;
$51 \%$ women), randomized patients in a factorial design to receive a low dose (1 g/day) of DHA+EPA, vitamin D3 (200 IU) supplementation, or placebo. ${ }^{7,56}$ After 5.3 years of follow-up, neither combination omega-3 fatty acids nor vitamin D3 was associated with a significantly lower incidence of major CVD events or invasive cancer. An analysis of major CVD events by sex subgroup, comparing the combination omega-3 fatty acid group with the placebo group, found that the HRs for major CVD events for women and men were 0.93 (95\% CI 0.76-1.15) and 0.91 (95\% CI 0.76$1.10)$, respectively ( $p=0.88$ for interaction).

The contrast between positive findings in JELIS for EPA in CVD risk reduction and subsequent negative studies of DHA+EPA combination omega-3 fatty acids suggests that EPA may have differential effects on CVD risk reduction. Insights regarding the effects of EPA in women can be gained from analyses of the Multi-Center, PlAceboControlled, Randomized, Double-B1INd, 12-week study with an open-label Extension (MARINE) and ANCHOR studies, which found that in women at elevated risk of CVD with $\mathrm{TG} \geq 500 \mathrm{mg} / \mathrm{dL}$ (MARINE) and $\geq 200$ to $<500 \mathrm{mg} / \mathrm{dL}$ (ANCHOR), icosapent ethyl $4 \mathrm{~g} /$ day significantly reduced TG in women by $21.5 \%$ and $22.7 \%$ in MARINE and ANCHOR, respectively, without an increase in LDL-C; these findings were generally comparable to findings in the overall study populations. ${ }^{57}$ Similar results were seen in a post hoc analysis of women with diabetes from the ANCHOR study. ${ }^{58}$ Changes in other atherogenic lipid, lipoprotein, and inflammatory parameters were favorable and generally consistent with results of the overall study that included women and men. ${ }^{57-63}$

\section{Reduction of Cardiovascular Events with Icosapent Ethyl-Intervention Trial}

The recently published REDUCE-IT study $(N=8,179$; $29 \%$ women) investigated CVD outcomes with icosapent ethyl $4 \mathrm{~g} /$ day, in addition to statin treatment in patients with elevated TG $(135-499 \mathrm{mg} / \mathrm{dL})$ who were $\geq 45$ years of age with established CVD or $\geq 50$ years of age with type 2 diabetes and at least one additional risk factor. ${ }^{9}$ Patients were randomized to icosapent ethyl or placebo. The primary composite endpoint (CVD death, nonfatal MI, nonfatal stroke, coronary revascularization, or unstable angina) occurred in significantly fewer patients in the icosapent ethyl group $(17.2 \%)$ compared with the placebo group $(22.0 \%)$ (HR $0.75 ; 95 \%$ CI $0.68-0.83 ; p<0.001$ ) after a median 4.9 years of follow-up (Fig. 2A). These data correspond to a number needed to treat to avoid one primary endpoint event of 21 (95\% CI 15-33).

Similarly, the key secondary composite endpoint (CVD death, nonfatal MI, or nonfatal stroke) occurred in $11.2 \%$ of patients in the icosapent ethyl group and $14.8 \%$ in the placebo group (HR 0.74; 95\% CI 0.65-0.83; $p<0.001$; Fig. 2B). The number needed to treat to prevent one key secondary endpoint was 28 (95\% CI 20-47). ${ }^{9}$

As reported in JELIS, the overall rate of coronary events was lower with women than with men. A subgroup analysis by sex in REDUCE-IT found that, in women, event rates for the primary endpoint were $13.3 \%$ in the icosapent ethyl arm and $15.6 \%$ in the placebo group (HR $0.82 ; 95 \%$ CI $0.66-1.01$ ), whereas for men, the event rates were $18.8 \%$ and $24.7 \%$ in the icosapent ethyl and placebo groups, respectively (HR 0.73; 

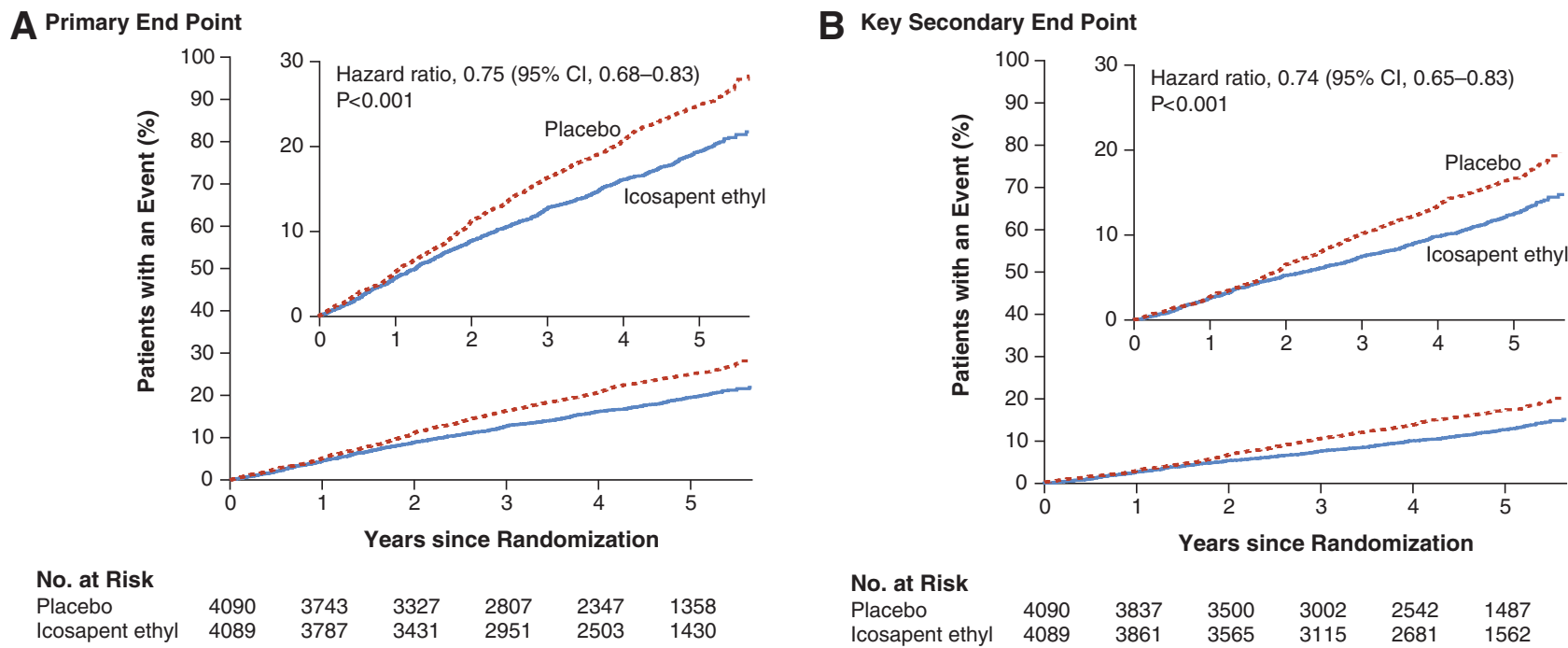

$\begin{array}{lllllll}\text { No. at Risk } & & & & & & \\ \text { Placebo } & 4090 & 3743 & 3327 & 2807 & 2347 & 1358 \\ \text { Icosapent ethyl } & 4089 & 3787 & 3431 & 2951 & 2503 & 1430\end{array}$

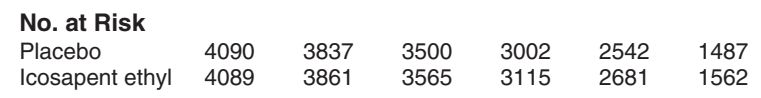

FIG. 2. Cumulative incidence of cardiovascular events in REDUCE-IT. ${ }^{9}$ Kaplan-Meier event curves for the primary efficacy endpoint, defined as a composite of CVD death, nonfatal MI, nonfatal stroke, coronary revascularization, or unstable angina in a time-to-event analysis (A); and the key secondary endpoint, defined as a composite of CVD death, nonfatal MI, or nonfatal stroke in a time-to-event analysis (B). In each panel, the inset shows the same data on an expanded y axis. The curves were visually truncated at 5.7 years because a limited number of events occurred beyond that time point; all patient data were included in the analyses. CVD, cardiovascular disease; MI, myocardial infarction; REDUCE-IT, Reduction of Cardiovascular Events with Icosapent Ethyl-Intervention Trial. From Bhatt et al., (C2019 Massachusetts Medical Society. ${ }^{9}$ Reprinted with permission from Massachusetts Medical Society.

95\% CI 0.65-0.82) (Table 2). The $p$-value for interaction by sex was not statistically significant $(p=0.33)$, suggesting that both women and men benefitted equally from treatment.

Comparable findings were reported for the key secondary endpoint when analyzed by sex (Table 2). Overall, the adverse events and serious adverse events in REDUCE-IT were low and similar to placebo. An issue that has been raised is that the placebo (mineral oil) was associated with small increases in LDL-C and high-sensitivity $\mathrm{C}$-reactive protein levels; however, an independent review suggested this could not explain the observed benefit associated with the intervention. ${ }^{64}$ Moreover, the CVD event rate in the placebo group was similar to that expected for comparable trials.

Based on the efficacy and safety profile demonstrated in REDUCE-IT, the United States Food and Drug Administra-

Table 2. Gender Differences in Primary AND SECONDARY ENDPOINTS IN REDUCTION of Cardiovascular Events WITH ICOSAPENT ETHYL-INTERVENTION TRIAL ${ }^{9}$

$H R(95 \%$ CI) $\quad$-Value for interaction

Primary endpoint: composite of cardiovascular death, nonfatal MI, nonfatal stroke, coronary revascularization, or unstable angina
Male
$0.73(0.65-0.82)$
$0.33^{\mathrm{a}}$

Female

$0.82(0.66-1.01)$

Key secondary endpoint: composite of cardiovascular death, nonfatal MI, or nonfatal stroke
Male
$0.72(0.62-0.82)$

Female

$0.80(0.62-1.03)$

\footnotetext{
${ }^{a}$ Interaction $p$-value not statistically significant, denoting comparable benefit.

$\mathrm{CI}$, confidence interval; HR, hazard ratio; MI, myocardial infarction.
}

tion recently approved icosapent ethyl for an expanded indication as an adjunct to maximally tolerated statin therapy to reduce the risk of MI, stroke, coronary revascularization, and unstable angina requiring hospitalization in adult patients with elevated TG levels ( $\geq 150 \mathrm{mg} / \mathrm{dL})$ and established CVD, or diabetes mellitus and two or more additional risk factors for CVD. ${ }^{65}$ Following suit, Health Canada recently approved the use of icosapent ethyl to reduce the risk of cardiovascular events (cardiovascular death, nonfatal MI, nonfatal stroke, coronary revascularization, or hospitalization for unstable angina) in statin-treated patients with elevated TG who are at high risk of cardiovascular events due to established CVD or diabetes and at least one other cardiovascular risk factor. ${ }^{66}$

In contrast to the positive findings in REDUCE-IT and JELIS for stable prescription EPA-only products, recent CVD outcome trials of combination omega- 3 fatty acids have not demonstrated reduced CVD risk. A differentiating factor between these clinical studies could be that combination omega-3 fatty acids contain DHA, which has been associated with increases in LDL-C, ${ }^{67,68}$ which may mitigate the beneficial effects in CVD risk reduction.

A number of ongoing studies will provide additional insights into the role of omega- 3 fatty acids in CVD risk reduction. ${ }^{69-71}$ The STRENGTH trial, which utilized $4 \mathrm{~g} /$ day of an EPA \pm DHA mixture, was recently stopped due to futility. ${ }^{72}$ Trial details should be forthcoming from the investigators; however, the lack of benefit of the combined EPA \pm DHA mixture is consistent with other combination omega-3 fatty acid trials, with published data showing lack of efficacy.

\section{Conclusions}

Women have unique barriers to the prevention of CVD. Underestimation of CVD risk in women may hinder appropriate preventive therapy. Women with CVD have been 
shown to have greater risk of adverse CVD outcomes than men, and women have a high risk of CVD associated with diabetes; therefore, application of evidence-based therapies in women is of paramount importance. Statins and other LDL-C-lowering treatments appear to have comparable benefits in high-risk women and men. In contrast, trials of treatments targeting other lipids have had mixed or negative results. The recently published REDUCE-IT study showed that addition of icosapent ethyl to statin treatment significantly reduced CVD events in patients at high risk of CVD, with comparable residual CVD risk reduction in women and men.

\section{Acknowledgments}

The genesis of this article was based on presentations from co-authors that took place during a Medical Advisory board supported by Amarin Pharma, Bedminster, NJ.

\section{Author Disclosure Statement}

L.M.: Consultant, Speaker's Bureau, Equity Investment: Amarin Pharma, Inc. Consultant: Livongo.

A.M.N.: Grant/research support: Amarin Pharma, Inc., Amgen, Regeneron, Sanofi, and Janssen; Consultant: Amarin Pharma, Inc., Amgen, Regeneron, Sanofi, Janssen, NovoNordisk, and AstraZeneca. Other financial or material support: NHLBI K01HL133416.

N.K.W.: No disclosures to report.

\section{Funding Information}

Medical writing assistance was provided by Peloton Advantage, LLC, an OPEN Health company, Parsippany, NJ, and supported by Amarin Pharma, Inc, Bedminster, NJ, USA.

\section{References}

1. Benjamin EJ, Muntner P, Alonso A, et al. Heart disease and stroke statistics-2019 update: A report from the American Heart Association. Circulation 2019;139:e56-e528.

2. Institute of Medicine Committee on Understanding the Biology of Sex and Gender Differences. The National Academies Collection: Reports funded by National Institutes of Health. Wizemann TM, Pardue ML, eds. Exploring the biological contributions to human health: Does sex matter? Washington, DC: National Academies Press (US), 2001.

3. Mosca L, Appel LJ, Benjamin EJ, et al. Evidence-based guidelines for cardiovascular disease prevention in women. American Heart Association scientific statement. Arterioscler Thromb Vasc Biol 2004;24:e29-e50.

4. Institute of Medicine. Women's health research: Progress, pitfalls, and promise. Washington, DC: The National Academies Press, 2010.

5. d'Emden MC, Jenkins AJ, Li L, et al. Favourable effects of fenofibrate on lipids and cardiovascular disease in women with type 2 diabetes: Results from the Fenofibrate Intervention and Event Lowering in Diabetes (FIELD) study. Diabetologia 2014;57:2296-2303.

6. The ACCORD Study Group, Ginsberg HN, Elam MB, et al. Effects of combination lipid therapy in type 2 diabetes mellitus. N Engl J Med 2010;362:1563-1574.

7. Manson JE, Cook NR, Lee IM, et al. Marine n-3 fatty acids and prevention of cardiovascular disease and cancer. N Engl J Med 2019;380:23-32.
8. The AIM-HIGH Investigators, Boden WE, Probstfield JL, et al. Niacin in patients with low HDL cholesterol levels receiving intensive statin therapy. N Engl J Med 2011;365: 2255-2267.

9. Bhatt DL, Steg G, Miller M, et al. Cardiovascular risk reduction with icosapent ethyl for hypertriglyceridemia. N Engl J Med 2019;380:11-22.

10. Humphries KH, Izadnegahdar M, Sedlak T, et al. Sex differences in cardiovascular disease-Impact on care and outcomes. Front Neuroendocrinol 2017;46:46-70.

11. Mosca L, Manson JE, Sutherland SE, Langer RD, Manolio T, Barrett-Connor E. Cardiovascular disease in women: A statement for healthcare professionals from the American Heart Association. Writing Group. Circulation 1997;96: 2468-2482.

12. Manson JE, Spelsberg A. Risk modification in the diabetic patient. In: Manson JE, Ridker PM, Gaziano JM, Hennekens $\mathrm{CH}$, eds. Prevention of myocardial infarction. New York, NY: Oxford University Press, 1996:241-273.

13. Hadi HA, Suwaidi JA. Endothelial dysfunction in diabetes mellitus. Vasc Health Risk Manage 2007;3:853-876.

14. Ghosh A, Gao L, Thakur A, Siu PM, Lai CWK. Role of free fatty acids in endothelial dysfunction. J Biomed Sci 2017;24:50.

15. Wang L, Manson JE, Forman JP, Gaziano JM, Buring JE, Sesso HD. Dietary fatty acids and the risk of hypertension in middle-aged and older women. Hypertension 2010;56: 598-604.

16. Gerber RT, Holemans K, O'Brien-Coker I, et al. Cholesterol-independent endothelial dysfunction in virgin and pregnant rats fed a diet high in saturated fat. $\mathrm{J}$ Physiol 1999;517 (Pt 2):607-616.

17. Vogel RA. Coronary risk factors, endothelial function, and atherosclerosis: A review. Clin Cardiol 1997;20:426-432.

18. Berger JS, Elliott L, Gallup D, et al. Sex differences in mortality following acute coronary syndromes. JAMA 2009;302:874-882.

19. Hochman JS, Tamis JE, Thompson TD, et al. Sex, clinical presentation, and outcome in patients with acute coronary syndromes. Global use of strategies to open occluded coronary arteries in acute coronary syndromes IIb Investigators. N Engl J Med 1999;341:226-232.

20. Bessesen DH, Cox-York KA, Hernandez TL, et al. Postprandial triglycerides and adipose tissue storage of dietary fatty acids: Impact of menopause and estradiol. Obesity (Silver Spring) 2015;23:145-153.

21. Karalis DG, Wild RA, Maki KC, et al. Gender differences in side effects and attitudes regarding statin use in the Understanding Statin Use in America and Gaps in Patient Education (USAGE) study. J Clin Lipidol 2016;10:833841.

22. Walsh MN, Joynt KE. Delays in seeking care: A women's problem? Circ Cardiovasc Qual Outcomes 2016;9(2 Suppl 1):S97-S99.

23. Mosca L, Benjamin EJ, Berra K, et al. Effectiveness-based guidelines for the prevention of cardiovascular disease in women-2011 update: A guideline from the American Heart Association. Circulation 2011;123:1243-1262.

24. St-Onge MP, Grandner MA, Brown D, et al. Sleep duration and quality: Impact on lifestyle behaviors and cardiometabolic health: A scientific statement from the American Heart Association. Circulation 2016;134:e367-e386.

25. Low CA, Thurston RC, Matthews KA. Psychosocial factors in the development of heart disease in women: Current 
research and future directions. Psychosom Med 2010;72: 842-854.

26. Mosca L, Linfante AH, Benjamin EJ, et al. National study of physician awareness and adherence to cardiovascular disease prevention guidelines. Circulation 2005;111:499-510.

27. Navar-Boggan AM, Peterson ED, D’Agostino RB, Sr., Pencina MJ, Sniderman AD. Using age- and sex-specific risk thresholds to guide statin therapy: One size may not fit all. J Am Coll Cardiol 2015;65:1633-1639.

28. Kannel WB, Sorlie P, McNamara PM. Prognosis after initial myocardial infarction: The Framingham study. Am J Cardiol 1979;44:53-59.

29. Bhatt DL, Roe MT, Peterson ED, et al. Utilization of early invasive management strategies for high-risk patients with non-ST-segment elevation acute coronary syndromes: Results from the CRUSADE Quality Improvement Initiative. JAMA 2004;292:2096-2104.

30. Yan AT, Yan RT, Tan M, et al. Management patterns in relation to risk stratification among patients with non-ST elevation acute coronary syndromes. Arch Intern Med 2007; 167:1009-1016.

31. Poon S, Goodman SG, Yan RT, et al. Bridging the gender gap: Insights from a contemporary analysis of sex-related differences in the treatment and outcomes of patients with acute coronary syndromes. Am Heart J 2012;163:66-73.

32. Donataccio MP, Puymirat E, Parapid B, et al. In-hospital outcomes and long-term mortality according to sex and management strategy in acute myocardial infarction. Insights from the French ST-elevation and non-ST-elevation Myocardial Infarction (FAST-MI) 2005 Registry. Int J Cardiol 2015;201:265-270.

33. Vaccarino V, Rathore SS, Wenger NK, et al. Sex and racial differences in the management of acute myocardial infarction, 1994 through 2002. N Engl J Med 2005;353:671682.

34. Canto JG, Rogers WJ, Goldberg RJ, et al. Association of age and sex with myocardial infarction symptom presentation and in-hospital mortality. JAMA 2012;307:813-822.

35. Nanna MG, Wang TY, Xiang Q, et al. Sex differences in the use of statins in community practice. Circ Cardiovasc Qual Outcomes 2019;12:e005562.

36. Peters SAE, Colantonio LD, Zhao H, et al. Sex differences in high-intensity statin use following myocardial infarction in the United States. J Am Coll Cardiol 2018;71:17291737.

37. Kim ES, Menon V. Status of women in cardiovascular clinical trials. Arterioscler Thromb Vasc Biol 2009;29:279_ 283.

38. Scott PE, Unger EF, Jenkins MR, et al. Participation of women in clinical trials supporting FDA approval of cardiovascular drugs. J Am Coll Cardiol 2018;71:1960-1969.

39. National Institutes of Health. NIH Policy and Guidelines on the Inclusion of Women and Minorities as Subjects in Clinical Research. 2001. Available at: https://grants.nih .gov/policy/inclusion/women-and-minorities/guidelines .htm Accessed January 31, 2020.

40. Mora S, Glynn RJ, Hsia J, MacFadyen JG, Genest J, Ridker PM. Statins for the primary prevention of cardiovascular events in women with elevated high-sensitivity C-reactive protein or dyslipidemia: Results from the Justification for the Use of Statins in Prevention: An Intervention Trial Evaluating Rosuvastatin (JUPITER) and meta-analysis of women from primary prevention trials. Circulation 2010; 121:1069-1077.
41. Ridker PM, Danielson E, Fonseca FA, et al. Rosuvastatin to prevent vascular events in men and women with elevated C-reactive protein. N Engl J Med 2008;359:2195-2207.

42. Gutierrez J, Ramirez G, Rundek T, Sacco RL. Statin therapy in the prevention of recurrent cardiovascular events: A sex-based meta-analysis. Arch Intern Med 2012;172:909919.

43. Taylor F, Huffman MD, Macedo AF, et al. Statins for the primary prevention of cardiovascular disease. Cochrane Database Syst Rev 2013:CD004816.

44. Fulcher J, O'Connell R, Voysey M, et al. Efficacy and safety of LDL-lowering therapy among men and women: Meta-analysis of individual data from 174,000 participants in 27 randomised trials. Lancet 2015;385:1397-1405.

45. Kato ET, Cannon CP, Blazing MA, et al. Efficacy and safety of adding ezetimibe to statin therapy among women and men: Insight from IMPROVE-IT (Improved Reduction of Outcomes: Vytorin Efficacy International Trial). J Am Heart Assoc 2017;6:e006901.

46. Cannon CP, Blazing MA, Giugliano RP, et al. Ezetimibe added to statin therapy after acute coronary syndromes. N Engl J Med 2015;372:2387-2397.

47. Sever PS, Gouni-Berthold I, Keech A, et al. Benefit of LDL-C lowering with evolocumab on cardiovascular outcomes by age \& sex: An analysis of the FOURIER trial [abstract 5002]. Eur Heart J 2018;39(suppl 1):1041-1042.

48. The FIELD Study Investigators, Keech A, Simes RJ, et al. Effects of long-term fenofibrate therapy on cardiovascular events in 9795 people with type 2 diabetes mellitus (the FIELD study): Randomised controlled trial. Lancet 2005; 366:1849-1861.

49. The HPS2-THRIVE Collaborative Group. Effects of extended-release niacin with laropiprant in high-risk patients. N Engl J Med 2014;371:203-212.

50. Yokoyama M, Origasa H, Matsuzaki M, et al. Effects of eicosapentaenoic acid on major coronary events in hypercholesterolaemic patients (JELIS): A randomised open-label, blinded endpoint analysis. Lancet 2007;369:1090-1098.

51. Rauch B, Schiele R, Schneider S, et al. OMEGA, a randomized, placebo-controlled trial to test the effect of highly purified omega-3 fatty acids on top of modern guidelineadjusted therapy after myocardial infarction. Circulation 2010;122:2152-2159.

52. ORIGIN Trial Investigators. n-3 Fatty acids and cardiovascular outcomes in patients with dysglycemia. N Engl J Med 2012;367:309-318.

53. The Risk and Prevention Study Collaborative Group. n-3 Fatty acids in patients with multiple cardiovascular risk factors: The Risk and Prevention Study Collaborative Group. N Engl J Med 2013;368:1800-1808.

54. ASCEND Study Collaborative Group, Bowman L, Mafham M, et al. Effects of n-3 fatty acid supplements in diabetes mellitus. N Engl J Med 2018;379:1540-1550.

55. D'Andrea E, Hey SP, Ramirez CL, Kesselheim AS. Assessment of the role of niacin in managing cardiovascular disease outcomes: A systematic review and meta-analysis. JAMA Network Open 2019;2:e192224.

56. Manson JE, Cook NR, Lee IM, et al. Vitamin D supplements and prevention of cancer and cardiovascular disease. N Engl J Med 2019;380:33-44.

57. Mosca L, Ballantyne CM, Bays HE, et al. Usefulness of icosapent ethyl (eicosapentaenoic acid ethyl ester) in women to lower triglyceride levels (results from the MARINE and ANCHOR trials). Am J Cardiol 2017;119:397-403. 
58. Brinton EA, Ballantyne CM, Guyton JR, et al. Lipid effects of icosapent ethyl in women with diabetes mellitus and persistent high tiglycerides on statin treatment: ANCHOR trial subanalysis. J Womens Health (Larchmt) 2018;27: 1170-1176.

59. Bays HE, Ballantyne CM, Kastelein JJ, Isaacsohn JL, Braeckman RA, Soni PN. Eicosapentaenoic acid ethyl ester (AMR101) therapy in patients with very high triglyceride levels (from the Multi-center, plAcebo-controlled, Randomized, double-blINd, 12-week study with an open-label Extension [MARINE] trial). Am J Cardiol 2011;108:682690.

60. Ballantyne CM, Bays HE, Kastelein JJ, et al. Efficacy and safety of eicosapentaenoic acid ethyl ester (AMR101) therapy in statin-treated patients with persistent high triglycerides (from the ANCHOR study). Am J Cardiol 2012; 110:984-992.

61. Bays HE, Ballantyne CM, Braeckman RA, Stirtan WG, Soni PN. Icosapent ethyl, a pure ethyl ester of eicosapentaenoic acid: Effects on circulating markers of inflammation from the MARINE and ANCHOR studies. Am J Cardiovasc Drugs 2013;13:37-46.

62. Ballantyne CM, Bays HE, Braeckman RA, et al. Icosapent ethyl (eicosapentaenoic acid ethyl ester): Effects on plasma apolipoprotein C-III levels in patients from the MARINE and ANCHOR studies. J Clin Lipidol 2016;10: 635-645.

63. Ballantyne CM, Bays HE, Philip S, et al. Icosapent ethyl (eicosapentaenoic acid ethyl ester): Effects on remnant-like particle cholesterol from the MARINE and ANCHOR studies. Atherosclerosis 2016;253:81-87.

64. FDA Briefing Document Endocrinologic and Metabolic Drugs Advisory Committee Meeting. 2019. Available at: https://www.fda.gov/media/132477/download Accessed January 31, 2020.

65. Vascepa [package insert]. Bridgewater, NJ: Amarin Pharma, Inc., 2019.

66. Vascepa [product monograph]. Etobicoke, Canada: HLS Therapeutics, 2019.

67. Wei MY, Jacobson TA. Effects of eicosapentaenoic acid versus docosahexaenoic acid on serum lipids: A systematic review and meta-analysis. Curr Atheroscler Rep 2011;13: 474-483.

68. Jacobson TA, Glickstein SB, Rowe JD, Soni PN. Effects of eicosapentaenoic acid and docosahexaenoic acid on lowdensity lipoprotein cholesterol and other lipids: A review. J Clin Lipidol 2012;6:5-18.

69. Budoff M, Brent Muhlestein J, Le VT, May HT, Roy S, Nelson JR. Effect of Vascepa (icosapent ethyl) on progression of coronary atherosclerosis in patients with elevated triglycerides $(200-499 \mathrm{mg} / \mathrm{dL})$ on statin therapy: Rationale and design of the EVAPORATE study. Clin Cardiol 2018;41:13-19.

70. Randomized trial for evaluation in secondary prevention efficacy of combination therapy-Statin and eicosapentaenoic acid UMIN000012069. UMIN Clinical Trials Registry 2016. Available at: https://upload.umin.ac.jp/ cgi-open-bin/ctr/ctr.cgi?function=brows\&action=brows \& recptno $=$ R000014051\&type $=$ summary\&language $=E$ Accessed January 31, 2020.

71. Nicholls SJ, Lincoff AM, Bash D, et al. Assessment of omega-3 carboxylic acids in statin treated patients with high levels of triglycerides and low levels of high density lipoprotein cholesterol: Rationale and design of the STRENGTH trial. Clin Cardiol 2018;41:1281-1288.

72. AstraZeneca ends cardiovascular outcomes study of Epanova as unlikely to show benefit [press release]. 2020. Available at: https://m.firstwordpharma.com/astrazenecaends-cardiovascular-outcomes-study-epanova-unlikely-showbenefit Accessed January 31, 2020.

Address correspondence to:

Lori Mosca, MD, MPH, PhD

Division of Cardiology, Department of Medicine Columbia university Irving Medical Center 51 Audubon Avenue

Suite 505

New York, NY 10032 Phone: 212-305-4752

USA

E-mail:1jm10@columbia.edu 